\title{
Paul Louis Rossi : un espoir renaissant
}

\author{
Roger-Michel Allemand \\ Laboratoire Babel - Université du Sud - Toulon - Var
}

Roger-Michel Allemand - Depuis novembre 2009, vous organisez avec Jacques Santrot et le Musée Dobrée de Nantes une exposition de sa collection de gravures et d'estampes d'Altdorfer, de Cranach, de Dürer et de ses élèves, de Georg Pencz et des frères Beham (Sebald et Barthold). Qu'est-ce qui vous fascine dans cet ensemble?

Paul Louis Rossi - Ce musée, pour des raisons romanesques, possède une fabuleuse collection de gravures. De nombreux Rembrandt, quatre-vingt-quatorze Dürer ainsi que des Lucas Cranach, Albrecht Altdorfer, et celui qu'Albrecht Dürer appelle le Prodigieux Lucas de Leyde. La collection est assez secrète, car vous le savez, on ne peut pas exposer en permanence les 
estampes et les gravures, et il semble miraculeux d'avoir l'occasion d'en montrer pour un temps très court quelques éléments. Il manque à cette collection Urs Graf, le peintre et graveur de Bâle. Très brièvement, il est le plus incroyable artiste de ce temps. Son œuvre est identique à celle de Jacques Callot: Les Malheurs de la guerre. Mais celle de Graf, un siècle auparavant, est encore plus sauvage, extravagante et folle. Vous comprendrez que c'est assez pour me préoccuper. On peut lire dans ces gravures tenues dans l'obscurité tout l'imaginaire, les rêves, les stratégies, les croyances des décennies qui succèdent au Quattrocento italien.

R.-M. A. - De la gravure à l'écriture, vous publiez parallèlement un texte sur l'histoire du Musée et sur cette collection, dans le numéro de mars 2010 de la revue Place publique. Toutefois, ce n'est pas que cela. Il s'agit d'une vision personnelle, forcément, mais aussi d'une sorte d'autoportrait indirect...

P. L. R. - Je verrais plutôt un portrait de la Ville. Un sentiment plutôt qu'une forme. Par exemple, un fragment du récit que je n'ai pas retenu. Du quai de la Fosse, celui du port et des docks, il existe une série d'impasses et de ruelles, les rues d'Ancin, des Cap-Horniers, Falconet, qui correspondent entre elles par des couloirs et des escaliers, et qui montent jusqu'à la rue de Flandres vers ce Musée Dobrée dont nous parlons. Vous voyez par là, dans cette ville grise, qu'il se produit presque toujours un incident, une découverte, une surprise qui change l'allure et la face des éléments. On pourrait en tirer un portrait de moi. Mais je suis beaucoup plus consciencieux qu'il ne paraît. Nullement aventurier, si l'on excepte l'aventure de l'esprit et celle, si vous le permettez, de la description qui change même la physionomie naturaliste des lieux et des histoires. 
R.-M. A. - Oui, mais je disais cela en pensant notamment au terme de "déambulation » que vous utilisez dans ce texte : la circulation dans les rues de Nantes, précisément, celle dans les couloirs de l'exposition, celle du regard sur les œuvres, ces sortes de coulées enfin, que nous apprennent-elles sur votre regard et sur la façon dont il dynamise et donne du mouvement à l'immobile?

P. L. R. - Évidemment, le mot déambulation résume ce que je viens d'énoncer, par sa longueur et le méandre de la prononciation. J'ai vraiment une stratégie de la randonnée, avec quelques principes sérieux. Partir d'un point précis par exemple. Ne jamais revenir sur ses pas. On a le droit seulement de croiser en boucle un segment usité de la dérive. Éviter les parcours trop connus, et périodiquement changer les itinéraires. Enfin, retenir la différence entre revenir dans le récit au point de départ, et l'idée de se rendre d'un point à un autre très différent dans son allure littéraire.

R.-M. A. - Comment ne pas songer aux déambulations oniriques $\mathrm{du}$ Buisson de datura? Dans Inscapes - ces paysages intérieurs - vous écrivez que les objets «sont le décor et le soutien des méditations romanesques» et évoquez «l'œil spirituel ». Le troisième œil de l'intuition ?

P. L. R. - Pour moi, cette notion d'Inscape est fondamentale (voir Rossi, 1994 et 2004a). Le mot est inventé par Gerard Manley Hopkins, que je considère comme le plus grand poète contemporain. C'est presque une provocation, pour éviter le terme «moderne », qui ne veut plus rien dire. Ce qu'il invente — Hopkins - dans la littérature évidemment, c'est la condensation dans le langage d'un événement, d'une image fugace, voire indescriptible, la forme d'un nuage, une vague 
particulière de la marée montante, l'éclosion d'un végétal. Cette fixation est mentale. Sa légitimité est inquiète. Ce ne sont pas les mots ni l'agencement qui décident. C'est le rapport intérieur du regardeur et du regardé. Le sentiment intérieur d'avoir atteint le même objectif que l'élément décrit dans le texte. Disons que le langage est pris comme une condensation du réel en mouvement.

R.-M. A. - Et la spéculation : quels rapports entre l'observation, l'imagination et le miroir?

P. L. R. - Je ne vois pas de spéculations dans l'écriture. Je serais intéressé par le spéculaire. Néologisme tardif en ce sens, très séduisant pour moi. La folie spéculaire, c'est-à-dire une sorte de frénésie, d'enchaînements aux limites de la logique. Par exemple, la plante nommée spéculaire, dite miroir de Vénus. Cependant, l'idée du miroir ne me trouble pas. Si vous voulez, je n'ai pas d'imagination, je déroule une sorte de scénario élémentaire et très précis. Le sens — s'il y a du sens — vient de lui-même, se manifeste pour le lecteur. Je dois avouer que je suis gaucher et que j'écris facilement de la droite vers la gauche, et que je puis lire le texte sans utiliser un miroir. Je pense sincèrement que le narcissisme - qui existe fatalement - nuit à la création. Je cherche vraiment à ne pas me regarder. J'aime au fond me lire comme l'expression écrite d'un étranger à moimême. Mon jugement peut être sévère.

R.-M. A. - Quelle est la place de l'analogie dans vos compositions? Je songe en particulier à «La Pensée analogique » dans La Rivière des cassis.

P. L. R. - Nous pensons avec Agnès Marcetteau, directrice de la Médiathèque de Nantes et du Musée Jules Verne, construire ce 
que j'appelle un Salon Analogique. C'est encore une Utopie. C'est dire que je suis intéressé par le système des analogies. Autrefois, je me souviens que nous appelions Jean-Pierre Faye, qui dirigeait la revue Change, Le Grand Analogue. Mais ma construction analogique est très rigoureuse. Elle n'accepte que des éléments déterminés qui ne nuisent pas au système. Il faudrait regarder du côté des Affinités électives, mais surtout étudier les monades et les théories du particulier et de l'universel dans la philosophie de Leibniz. C'est l'idée de l'escalier qui me retient. Je vois cela comme un chemin initiatique qui monte vers une suite imaginaire. Bien entendu, nous sommes aux premières marches. Il faut inclure la magie dans les éléments de base.

R.-M. A. - Que vous ayez intitulé le texte pour la Folie Dobrée «La Demeure irlandaise » ne me semble pas fortuit: il y a de l'épiphanie chez vous. Joyce, que vous évoquez notamment dans le Buisson, vous a-t-il influencé de ce point de vue ?

P. L. R. - Voilà bien la singularité de cette ville hanséatique, autrefois l'un des premier port de l'Europe, emplie de fantômes, convoitée par les Vendéens royalistes, éprise des Lumières, emplie de corsaires, de marchands hollandais et flamands, de Portugais et d'Italiens, où le Baron de La Hontan dispute avec un médecin portugais du Consentement universel avant de s'embarquer vers le Canada. Avec cette famille Dobrée, négociants, marins, quelquefois pirates, protestants qui appellent leur construction, vers 1828, Le Manoir des Irlandais. J'aimerais évidemment que Nantes soit pour moi comme la ville de Dublin pour James Joyce. Mais je partage mon sentiment avec Venise, que je connais depuis mon enfance. Cette notion d'épiphanie, très proche de la notion d'inscape, est très difficile à 
cerner, car appartenant justement à Joyce, écrivain mythique. Il tire la notion d'épiphanie vers la prose, la banalité, le vulgaire, la dépréciation, et même la mauvaiseté. Je dois être capable de violence littéraire et polémique, mais pas de vraie méchanceté. C'est un défaut. Je viens de revoir le dernier film de John Huston, composé pour les Gens de Dublin: Dubliners: le spectacle entier est pris et réalisé dans le sens sublime d'une épiphanie de cette nuit irlandaise sous la neige d'hiver.

R.-M. A. - Le temps, la durée, doit jouer un rôle très important dans votre œuvre.

P. L. R. - Toute œuvre est une sorte de combat mené contre le temps et la durée. Du moins ce qu'on appelle le temps. Je ne suis pas différent des autres écrivains. Ma singularité serait de me situer dans un espace-temps qui ne tient pas compte fatalement des intervalles et des classifications: passé, présent, futur. D'une certaine façon, je ne respecte ni la chronologie ni la concordance des temps. Mais ce qui est au fond de vos questions, je le vois comme une autre chose dont nous parlerons par la suite, qui est le fonctionnement de la mémoire et de l'oubli.

R.-M. A. - Serait-il faux de dire que vos différentes activités d'écrivain - poète et romancier, critique d'art, de cinéma, de jazz - participent d'une même démarche, disons philosophique?

P. L. R. - À la vérité, très jeune, je voulais être journaliste, et j'estimais de mon devoir d'écrire quand on me le demandait. C'est ainsi que j'ai commencé la critique cinématographique dans un petit journal provincial. Plus tard, à Paris, j'écrivais dans Jazz Magazine, mais je me suis aperçu au bout d'un temps 
que je n'avais plus le désir de rédiger mes chroniques, et que je devenais désagréable. J'ai ainsi compris que la tâche littéraire est incompatible avec l'exténuant travail journalistique. Mais je continue d'écrire des articles. Je viens de publier dans la revue Europe un long texte pour les deux volumes des Écrits mémorables de Louis Massignon. Revenons à la démarche. Il est évident que je voulais, à l'origine, construire une Esthétique. Cependant, il eût fallu un temps considérable d'élaboration, un soutien matériel et une étendue philosophique universelle. Mais j'accumule patiemment des notes et des essais, sur la peinture en particulier, qui finiront sans doute par produire une synthèse philosophique.

R.-M. A. - Je sais que ma question est probablement démesurée, mais quel est le fil conducteur dans ce labyrinthe?

P. L. R. - Je serais tenté de dire : il n'y a pas de fil. Je crois que le système interdit les amalgames impossibles. Ma critique de l'esthétique ne prétend pas tout embrasser, elle est très sélective. Pour prendre un exemple, il y a des peintres célèbres dont je ne parle pas, et pour mes contemporains, avec qui je ne souhaite pas travailler. Ils peuvent être très bons, mais ils ne figurent pas dans ma cosmogonie. Il m'arrive cependant de changer d'avis. Si vous voulez, je ne suis pas un ogre ni un fanatique ; d'une certaine façon, j'ai déjà beaucoup à faire avec ce qui me convient.

R.-M. A. - Reste la question du labyrinthe: ne s'agirait-il pas d'échapper à la ligne invisible évoquée par Borgès ? Entre Le Livre de sable (Borgès, 1975) et "La Bibliothèque de Babel» (Borgès, 1957), où en êtes-vous de vos états provisoires? 
P. L. R. - J'ai beaucoup fréquenté Jorge Luis Borgès. Je me suis intéressé à son ouvrage d'une Histoire de l'infamie (2001). Mais si vous voulez, je n'ai pas la prétention encyclopédique. Et je tiens vraiment à choisir mes objets. L'Histoire des Royaumes celtiques, par exemple, et celle des sôteria - stèles opisthographes de la Grèce archaïque en l'honneur de Zeus Sôter et de la victoire sur les Galates - deux ensembles recueillis dans Les États provisoires. Je suis préoccupé sans doute par la notion d'identité, voire de légitimité. Ne pas oublier que mon père était un Italien du Veneto, et que ma mère venait de la Cornouaille bretonne. Mes grands-parents Le Queffelec parlaient devant moi une langue gaëlique. Mais le Provisoire corrige cette ambition. Sur le plan poétique, j'en resterai certainement à cette suite de développements légendaires.

R.-M. A. - À simplement énoncer le retour du mot «nuit» dans certains de vos ouvrages (Rossi, 1958, 1997, 1998, 2000), on se dit qu'il doit bien être question de sortir de l'obscurité.

P. L. R. - J'avais cet ami, le peintre Gaston Planet, qui disait : «D'abord je veux savoir ce qu'est la nuit. Nuit mon mot préféré ». Il est vrai que mon premier livre de poésies s'intitule Liturgie pour la Nuit. Je découvrais la nuit moderne des villes Nantes et Paris - comme Louis Aragon dans sa description de la nuit au parc des Buttes-Chaumont (voir Aragon, 1973, chapitre 2) : "La nuit a des sifflets et des lacs de lueurs. Elle pend comme un fruit au littoral terrestre, comme un quartier de bœuf au poing d'or des cités. » Mais je suis moi-même surpris du nombre de cette expression de nuit dans le titre de mes livres. Je ne crois pas que je cherche à m'en échapper. Il faut prendre cet usage plutôt comme le symptôme d'une inquiétude, 
d'une partie sombre de moi-même que je cherche à décrire et à exprimer.

R.-M. A. - À l'occasion d'une autre exposition, aux musées des Beaux-Arts de Nantes, vous vous peigniez en Visiteur du clair et de l'obscur...

P. L. R. - Il m'est arrivé plusieurs fois de visiter seul des musées la nuit. À Leipzig par exemple, il y avait une peinture de Nolde au bout d'une sorte de corridor. C'est un privilège incomparable. J'ai un grand souvenir de cette exposition du Musée des Beaux-Arts de Nantes, en septembre 2004. J'étais chargé de dépoussiérer — c'est le mot - le musée. Évidemment, je n'ai touché à rien, ou presque. J'ai travaillé avec les réserves, ce continent englouti de tous les musées, et fait restaurer quelques tableaux. Et surtout, j'ai placé dans chacune des salles des œuvres de peintres contemporains, en contrepoint : Viallat, Planet, Jean-Michel Meurice, Shirley Jaffe. Et même dans la salle des primitifs italiens, une sculpture de fougère géante des Vanuatu prêtée par Madame Marie-Hélène Santrot. Le curieux de l'histoire, si l'on ne connaît pas le musée, c'est que l'on ne pouvait pas s'apercevoir des transformations. C'est dire que je suis contre une distraction, une esthétisation de l'Art. Je pourrais dire ceci : Ne rien ajouter, l'Art consiste à supprimer.

R.-M. A. - Dans les Aventures du baron de La Hontan, dont le texte n'est pas encore publié dans son intégralité, vous évoquez les méandres de la Rivière Longue : métaphore de l'articulation entre le réel et l'invention?

P. L. R. - On remarquera que j'écris La Hontan, et non Lahontan. C'est La Fontaine en béarnais. Je crois avoir entrepris une 
réhabilitation du Baron. Explorateur du Québec et du système de communication des Lacs. Inventeur du Sauvage de Bon sens et qui a de l'Esprit. Défenseur de la liberté des femmes. Ami de Leibniz et de la reine Sophie-Charlotte du Brandebourg. Inspirateur de Diderot et de Jean-Jacques Rousseau. J'en oublie certainement. Il décrira son expédition, jusqu'à l'arrivée de l'hiver, appelée de La Rivière Longue. Il est impossible de dérouler ici les méandres de l'expédition. Mais je l'ai comparée à celles de Gulliver, aux randonnées d'Antonin Artaud au Pays des Tarahumaras, ou bien encore à celles d'Henri Michaux décrites dans Ecuador (1990).

R.-M. A. - Et La Rivière des cassis ne fournit-elle pas une clé fondamentale : celle de la rêverie lexicale?

P. L. R. - Après avoir publié le live de La Rivière des cassis, je suis allé, avec mon ami le peintre André Lambotte, explorer la sinueuse Semoy: Rivière de Cassis, selon Rimbaud (1999, p. 148-149). Le singulier est qu'il s'agit sans doute d'un anglicisme pour Cathel, et surtout qu'on ne possède aucune étymologie sérieuse du mot cassis lui-même. Il faut lire l'argumentaire du livre. J'ai d'ailleurs eu la remarque d'une amie, docteur en pharmacie, qui prétendait corriger le titre du premier chapitre, Ribesées, en ribésiées, nom de famille des groseilles. Ribesée est bien entendu un néologisme qui désigne l'abus de consommation des cassis mélangés à plusieurs alcools, en souvenir des beuveries de Rabelais, qui doit écrire le mot pour libations - libesiées ou libesées - et autres billevesées de sa langue merveilleuse.

R.-M. A. - Le Voyage de sainte Ursule, Le Potlach, les Aventures du baron de LaHontan: de l'exploration et de l'expédition comme contrepoints à une introspection? 
P. L. R. - À la vérité, je me vois plutôt comme un émigrant. Un spécialiste d'explorations étranges. Bien entendu, cela passe aussi dans le vocabulaire. Mais identique au terme de nuit, on peut considérer cela comme un symptôme que je ne comprends pas tout à fait. Je me définis souvent comme un non-voyageur, je dis qu'il n'y a rien à voir dans le Monde. J'ajoute il est vrai : il n'y a rien à voir si vous ne regardez rien. C'est ainsi que je peux aller très loin pour vérifier un détail, et que je reprends parfois des itinéraires anciens, comme celui de Saumur et de la Prison Centrale de Fontevrault dans Le Vieil homme et la Nuit.

R.-M. A. - Lorsque vous parlez de Nantes comme de La Voyageuse immortelle, c'est donc de vous que vous parlez: un voyageur immobile - ou même une sorte d'île?

P. L. R. - La Voyageuse immortelle est vraiment une figure de proue, entre la Fiancée du pirate et sainte Anne. Il existe à Nantes une Butte Sainte Anne, dernier soubresaut des schistes rouges roses du Massif armoricain, qui domine le port et l'estuaire, avec une statue de la sainte en haut des marches. Les rues portent des noms de corsaires. Il y a même une femme, Julienne David, prisonnière des Anglais durant la Révolution. Tout en haut, on trouve le Musée Jules Verne et le Planétarium. Cette ville cosmopolite suscite des vocations de navigateurs, d'explorateurs et d'aventuriers. Il est vrai que j'ai une affection pour les îles. L'île d'Yeu en particulier, où j'ai composé les poèmes cosmiques d'Élévation Enclume. Avec le Japon, c'est plus compliqué. J'ai écrit pour Jean-Michel Meurice, qui est un grand voyageur, un nô de poche intitulé Le Pont suspendu, et je dois aussi travailler à présent sur la poésie des haïkus et des tanka pour un colloque à Lyon. Il est évident que la situation des îles provoque au niveau artistique un mystère. Je suis 
encore stupéfait par l'incroyable mise en scène gestuelle et musicale du nô.

R.-M. A. - Quand je pense à la prégnance de l'étrange ou du fantastique dans votre œuvre (Rossi, 1962, 1993, 1998, 2002, 2006), je me demande si, en fait, vous ne dialoguez pas avec les morts.

P. L. R. - J'ai souvent expliqué que l'on pouvait considérer certains de mes récits comme des rêves. Le roman de La Villa des chimères, bien entendu, avec un chapitre que personne ne semble avoir lu, et qui met en scène - dans un cloître - les écrivains maudits qui se déchirent. Ainsi que Les Nuits de Romainville, avec le fantôme de Gérard de Nerval qui cherche dans la nuit la route de Meaux et le chemin de l'Allemagne. Sans doute, je vis en partie avec des ombres, beaucoup plus nombreuses que je ne le laisse paraître. Mais mon dialogue avec les morts n'est pas apaisé. Je continue de critiquer leurs actions et de leur faire des querelles pour des détails qui me hantent. J'ai écrit dans Les États provisoires un texte intitulé «Stèle des mots et des morts », qu'un jeune musicien, Grégoire Lorieux, vient de mettre en scène et musique. L'exercice de la littérature représente, pour certains sujets, un objet sérieux et probablement une consolation.

R.-M. A. - Nous parlons d'images, d'imaginaire, d'imagination : quels rapports avec vos exercices oulipiens des Inimaginaires?

P. L. R. - Je crois que j'aimerais ne pas avoir d'imagination. C'est pourquoi j'étais très heureux de ces exercices des Inimaginaires, initiés par Jacques Roubaud, qui nous ont donné une sorte de détente, au sens du tempo comme dans le jazz, une rapidité, un traitement acrobatique du langage, et qui nous ont engagés 
dans l'élaboration et la compositions en commun. En particulier l'utilisation de la technique des renga sur le mode japonais. Cependant, je n'ai jamais participé aux exercices de l'Oulipo, et j'ai écrit dans le Vocabulaire de la modernité littéraire une chronique qui exprime quelques réserves. Ma crainte, avec le temps, est de voir la scène occupée par des répétitions et surtout, outre l'usure du temps, par une suprématie de moyens mécaniques de compositions qui introduisent une technicité douteuse dans l'écriture. Une croyance dans l'efficacité de la machine. Mais d'une certaine façon, ce n'est pas mon objet.

R.-M. A. - Vous étiez l'ami de Perec, vous avez collaboré aux Lettres françaises et à la revue Change, entre autres, et avez affirmé «qu'il était temps, concernant la Poésie (et la Littérature), d'interrompre cette sorte de fuite en avant qui caractérise l'Art de notre temps, et qui ne vise qu'à précipiter la destruction des formes - de l'intellect, et de la création. Il est temps [...] d'interroger à nouveau l'esthétique (et donc, la politique) et de tenter une définition neuve de la modernité. » (Rossi, 2000, prière d'insérer). Quelles sont aujourd'hui vos conclusions à ce propos?

P. L. R. - J'étais très ami avec Georges Perec. Il me poussait à publier des livres. Je manquais de temps pour négocier, mais j'avais écrit dans Libération un texte intitulé «Les Langues minoritaires", et je n'avais pas compris que nous avions, Georges et moi, la même expérience, enfants, de vivre durant une guerre dans une campagne éloignée des villes. C'est pourquoi le texte commençait par cette phrase : «J'ai vécu dans les temps paléolithiques». Je ne crois pas que l'on puisse stopper le mouvement de la modernité. Mais j'ai une affection particulière pour l'essai de Sigmund Freud, Malaise dans la 
civilisation. Il faut conserver ce titre qui montre très bien que la superstition du progrès inéluctable ne peut créer que du déceptif. Aujourd'hui, en ce qui me concerne, je suis consterné par l'inflation d'un art décadent dans la peinture et par le système répétitif des Installations : c'est-à-dire un art tombé dans son idéologie. Le vieil art moderne en vérité de la marchandise, du commerce et de la propagande.

R.-M. A. - N'est-ce pas que votre œuvre, au fond, place la recherche de la Beauté au-dessus de tout? Qu'elle témoigne d'une foi inébranlable en ses vertus?

P. L. R. - Oui, je serais d'accord avec vous : la beauté par-dessus tout. On m'avait demandé une fois mon appréciation sur une exposition de peintures d'amateurs, dans une entreprise. J'avais répondu: Ça serait beau si ça n'était pas laid. Grand succès parmi les employés, qui se servaient de l'expression. Vous ne pouvez pas parler de la musique si vous n'entendez pas la musique. Idem avec la peinture : un nombre incroyable de gens - la gente - prennent le tableau pour un spectacle qui raconte une histoire. Le problème de l'esthétique est que le philosophe peut ne rien comprendre à la littérature; Hegel par exemple, qui prononce des inepties à propos du théâtre de Schiller. Il faudrait relire Kant, La Critique de la raison pure. Le passage sur la Schwärmerei, la folie enthousiaste. Le mot est relevé par Baudelaire quand il parle de la beauté. On a dit que j'étais millénariste. C'est-à-dire que je croyais à une rédemption du temps à la suite des mille ans accomplis. C'est l'objet de mon introduction du Colloque de nuit. Supériorité de l'oiseau des îles qui construit en guise de séduction un léger monticule avec des graines noires et les élytres brillants du scarabée. 
R.-M. A. - Altdorfer, Dürer...: la dimension alchimique est perceptible dans leurs œuvres. Vous me direz que c'est un lieu commun ésotérique de la Renaissance, au point que la salamandre était le chiffre de François 1er. Mais à vous lire, je vous placerais volontiers sous cet emblème aussi. Aurais-je tort?

P. L. R. - Je suis agnostique, c'est-à-dire non préoccupé par les fins dernières. Par contre, j'ai une sympathie pour Duns Scot et pour l'Animisme, plus proches de la nature. L'art est sans doute une alchimie compliquée. Si vous voulez, la magie me sert de support et de défense, avec une part d'humour. Je suis heureux que vous parliez de François $1^{\mathrm{er}}$ et de la Salamandre. C'était la petite chaufferette verte de mon grand-père menuisier, où il faisait fondre la gomme arabique. C'est un animal aussi, proche des lieux humides et des ruisseaux, qui a la réputation de survivre dans le feu : Esprit du feu - esprit du nitre sublimation - nutrisco et estingo: soit J'entretiens et j'éteins. Vous remarquez à quelle rapidité je suis entraîné par le courant analogique.

R.-M. A. - Terminons, si vous le voulez bien, par une sorte de retour au point de départ de notre périple: La Traversée du Rhin, et du Danube (Rossi, 2009) - le dialogue avec l'Allemagne - , que vous dit-il de ce pays et de notre civilisation européenne?

P. L. R. - Ce livre de La Traversée du Rhin est douloureux et chaotique. Il est à sa parution donné comme un exemple de la modernité littéraire. Il n'était pas simple pour moi de franchir le Rhin. J'ai depuis ce temps beaucoup fréquenté les villes 
allemandes: Francfort, Hambourg, Leipzig, Berlin, Munich. D'une certaine façon, je suis un Européen de nature. Mais je suis politiquement un Européen convaincu. Je veux dire un citoyen de la culture et civilisation européenne. La civilisation européenne a toujours existé, depuis son origine. Depuis la Grèce antique, la domination des Celtes et des Romains. Il est sans doute temps de la réhabiliter. C'est-à-dire de comprendre que l'Europe ne peut pas exister seulement au niveau économique, industriel et commercial. Il faut absolument s'occuper des langues européennes, universelles et minoritaires. Et de tout ce qui unit les Européens. Je pense encore aux gravures de Dürer, d'Albrecht Altdorfer et d'Urs Graf, je pense au Prodigieux Lucas de Leyde. Il faudrait que nous puissions espérer, et retrouver l'esprit de la Renaissance et de la philosophie des Lumières.

\section{Bibliographie}

Aragon, Louis. 1973, Le Paysan de Paris, Paris, Gallimard, coll. « Folio ».

BORGÈs, Jorge Luis. 1957, Fictions, traduit de l'espagnol par Paul VERDEVOYE et IBARRA, Paris, Gallimard, coll. « Folio »;

—. 1975, Le Livre de sable, traduit de l'espagnol par Françoise RosSET, Paris, Gallimard, « Folio »;

—. 2001, Histoire universelle de l'infamie. Histoire de l'éternité, traduit de l'espagnol par Laure GuILLE-BataILLON, Paris, Christian Bourgois. 
FREUD, Sigmund. 2009, Le Malaise dans la civilisation, traduit de l'allemand par Bernard LORTHOLARY, Paris, Seuil, coll. « Points ».

GoETHE, Johann Wolfgang von. 1993, Les Affinités électives, traduit de l'allemand par Joseph-François AngELLOZ, Paris, Flammarion, coll. « GF ».

JoyCE, James. 1993, Gens de Dublin, traduit de l'anglais par Benoît TADIÉ, Paris, Flammarion, coll. « GF ».

KANT, Emmanuel. 2001, Critique de la raison pure, traduit de l'allemand par Alain RENAUT, Paris, Garnier Flammarion, coll. «GF ».

MASSIGNON, Louis. 2009, Écrits mémorables, Paris, Robert Laffont, coll. « Bouquins », 2 vol.

MichauX, Henri. 1990, Ecuador, Paris, Gallimard, coll. « L'Imaginaire ».

Rimbaud, Arthur. 1999, Poésies. Une saison en enfer. Illuminations, Paris, Gallimard, coll. « Folio ».

RossI, Paul Louis. 1958, Liturgie pour la nuit, Paris, Millas Martin;

—. 1962, Silence et plainte, Dijon, Chambelland;

-. 1970, Élévation Enclume, dessins de Gaston Planet, Beauvoir-sur-Mer, Imprimerie du Marais, réédité en 1997 par Le Temps qu'il fait;

—.1973, Le Voyage de sainte Ursule, Paris, Gallimard;

-. 1975, Inimaginaires, en collaboration avec Pierre LARTIGUE, Lionel RAY, Jacques RouBAUD, Beauvoir-sur-Mer, Imprimerie du Marais; 
—. 1979, Le Potlach, Paris, Hachette-P.O.L;

—. 1981, La Traversée du Rhin, Paris, Hachette-P.O.L;

—. 1984, Les États provisoires, Paris, P.O.L;

-. 1993, L'Ouest surnaturel, Paris, Hatier;

-. 1994, Inscapes, avec des dessins de François DILASSER, Cognac, Le Temps qu'il fait;

—. 1996, Vocabulaire de la modernité littéraire, Paris, Minerve;

—. 1997, Le Vieil homme et la Nuit, Paris, Julliard;

-. 1998, Les Nuits de Romainville, avec des photographies de Jean-Pierre Colin, Cognac, Le Temps qu'il fait;

—. 2000, Le Colloque nuit, en collaboration avec Philippe BECK et Yves DI Manno, Cognac, Le Temps qu'il fait;

—. 2001, La Voyageuse immortelle, Cognac, Le Temps qu'il fait;

—. 2002, La Villa des chimères, Paris, Flammarion;

-. 2003, La Rivière des cassis, avec des dessins de Marie-Claude BuGEAUD, Nantes, joca seria;

—. 2004a, Paysage intérieur, inscape, Nantes, joca seria;

—. 2004b, Visiteur du clair et de l'obscur, Nantes, joca seria;

—. 2005, Visage des nuits, Paris, Flammarion;

—. 2006, Le Buisson de datura, Nantes, joca seria;

-. 2009, Vies d'Albrecht Altdorfer, peintre mystérieux du Danube, Paris, Bayard. 